\title{
Advanced lung disease conference
}

Dr. David Sugarbaker has often been described as a force of nature. His passion for clinical excellence drove him to establish the modern day paradigm for thoracic surgical education in the United States and abroad. Dr. Sugarbaker advanced the field of invasive interventions for mesothelioma and other thoracic malignancies and established world premier programs at the Brigham \& Women's Hospital in Boston and, more recently, at Baylor St. Luke's Medical Center in Houston. He was driven to continue the advancement of the management and treatment for advanced lung diseases as evidenced by his initiation of Baylor College of Medicine's first ever Lung Institute. The Advanced Lung Disease Conference was in many ways a vision of Dr. Sugarbaker in collaboration with the transplant program, to enhance awareness of the diagnosis and treatment of a variety of end-stage lung diseases. In fact, Dr. Sugarbaker was instrumental in the development of the lung transplant program at Brigham and Women's Hospital, which continues to thrive.

The current journal focuses on evolving trends in the management and treatment of advanced lung disease. There are several disease categories that are covered under the broader umbrella of advanced lung disease. These include idiopathic pulmonary fibrosis (IPF) and the broader field of interstitial lung disease, pulmonary arterial hypertension (PAH), chronic obstructive pulmonary disease (COPD), severe asthma, and cystic fibrosis (CF). In addition, there is the generic heading of end-stage lung disease which can include many of these diseases, but can also progress through multiple pathways including infectious diseases. End-stage lung disease may be acute or chronic but very often permanent. Chronic lower respiratory disease is the fourth leading cause of death in the United States according to the centers for disease control and prevention (CDC) (1). The treatment for end-stage lung disease depends on the spectrum of the disease process. It may include acute intervention such as extracorporeal membrane oxygenation (ECMO), or advanced medical therapies such as gene therapies, stem cell therapies, supportive care, palliative care and lung transplantation.

According to World Health Organization estimates, COPD claimed the lives of 3 million people in 2016 making it the $3^{\text {rd }}$ leading cause of death in the world (2). The successful treatment of COPD requires timely diagnosis and methodical treatment plans, most often with the use of combined inhalers and/or oral or systemic anti-inflammatory therapies. In its most advanced form, lung transplantation is emerging as an important option for patients suffering from end-stage COPD (3). Similarly, CF also requires timely detection and lifelong surveillance and adherence to strict medical antibiotic prophylactic and supportive therapies. Patients with IPF can deteriorate very rapidly without transplant. If this occurs, they may become too ill to benefit from transplantation, thus patients with IPF require early discussions about transplantation to ensure their candidacy if and when lung transplantation is required. $\mathrm{PAH}$ is a disease of the small and large vessels of the lung. There are subtypes of $\mathrm{PAH}$, and it may also be secondary to inherent parenchymal disease. When patients develop pulmonary hypertension secondary to diseases such as COPD or IPF, this can have cascading effects on the heart and other organ systems in the body. Similarly, PAH often affects the right side of the heart leading to right ventricular dysfunction and systemic consequences. Asthma is a disease process that also falls under "advanced lung disease" but rarely leads to transplantation. Severe asthma is managed with lifestyle modifications as well as inhaler and more recently biological therapies.

There are several additional topics covered in the ensuing issue which will include specific aspects of lung transplantation: preoperative evaluation and selection, intraoperative considerations, surgical techniques, innovations such as ex vivo lung perfusion (EVLP), and utilization of ECMO in perioperative management. Lung transplant has become the gold standard for patients with end-stage lung disease secondary to a number of diseases mentioned above. Although lung transplantation is not the right therapy for everyone, it is an important one for those who are candidates. It is helpful to understand when a patient is a candidate and when they are not so that this option can be kept available for as many patients as possible. Additional topics that are covered include the logistics of donor management and the logistics of travel and transportation for a lung transplant.

We are confident that you will enjoy and find useful this focused edition on advanced lung diseases in the fournal of Thoracic Diseases (FTD). We hope to highlight relevant trends for your academic growth, but more importantly, we hope this focused edition improves your clinical practice and enhances your care of patients with advanced lung disease. 


\section{Acknowledgments}

None.

\section{Footnote}

Conflicts of Interest: Dr. Loor receives grant support from Transmedics (Andover, MA) for his involvement in Ex Vivo Lung Perfusion clinical trials and has received grant support within the past three years by Gentige for development of an ECMO registry and United Therapeutics for translational EVLP research. The other authors have no conflicts of interest to declare.

Ethical Statement: The authors are accountable for all aspects of the work in ensuring that questions related to the accuracy or integrity of any part of the work are appropriately investigated and resolved.

\section{References}

1. Heron M. Deaths: Leading Causes for 2016. Natl Vital Stat Rep 2018;67:1-77.

2. Global Health Estimates 2016: Deaths by Cause, Age, Sex, by Country and by Region, 2000-2016. Geneva: World Health Organization; 2018.

3. Thabut G, Mal H. Outcomes after lung transplantation. J Thorac Dis 2017;9:2684-91.

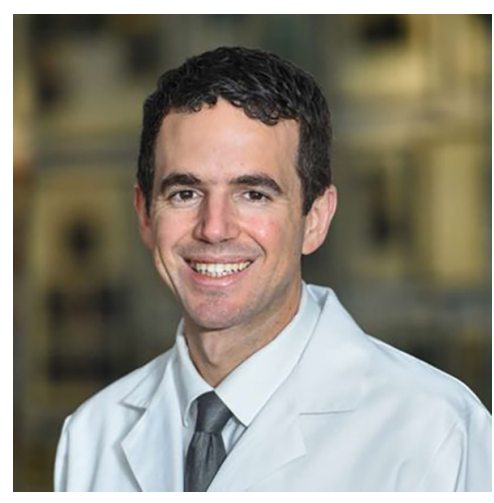

Gabriel Loor

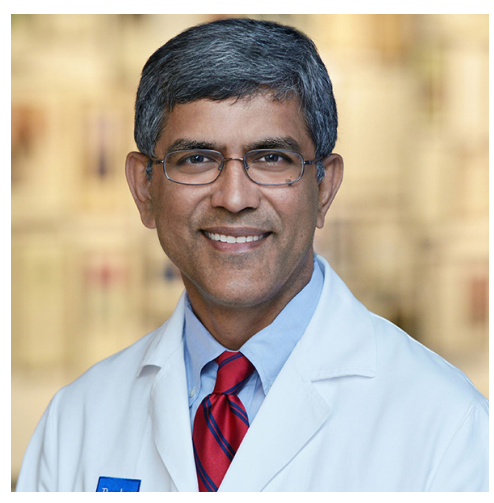

Prasad Manian

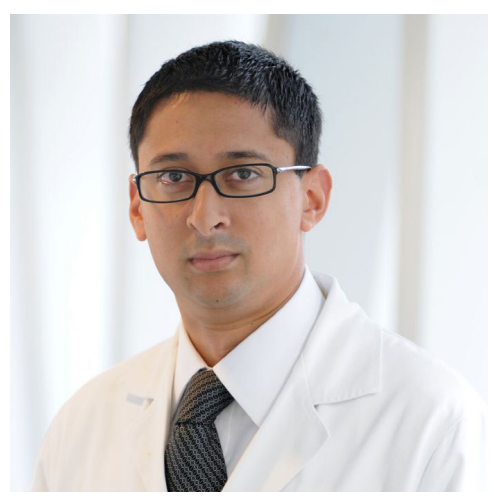

Amit Parulekar

Gabriel Loor, MD

Michael E. Debakey Department of Surgery, Division of Cardiothoracic Transplantation and Circulatory Support, Baylor College of Medicine, Houston, TX, USA. (Email: Gabriel.Loor@bcm.edu)

Prasad Manian, MD

Department of Medicine, Division of Pulmonary and Critical Care Medicine, Baylor College of Medicine, Houston, TX, USA.

(Email:pmanian@bcm.edu)

Amit Parulekar, MD

Department of Medicine, Division of Pulmonary and Critical Care Medicine, Baylor College of Medicine, Houston, TX, USA.

(Email: paruleka@bcm.edu)

doi: $10.21037 /$ jtd.2019.03.37

View this article at: http://dx.doi.org/10.21037/jtd.2019.03.37

Cite this article as: Loor G, Manian P, Parulekar A. Advanced lung disease conference. J Thorac Dis 2019;11(Suppl 14):S1686-S1687. doi: 10.21037/jtd.2019.03.37 